\title{
Neurophysiological Characterization of Thalamic Nuclei in Epileptic Anaesthetized Patients
}

\author{
Lorena Vega-Zelaya ${ }^{1}$, Cristina V. Torres ${ }^{2}$, Marta Navas ${ }^{2}$ and Jesús Pastor ${ }^{1, *(\mathbb{C})}$ \\ 1 Department of Clinical Neurophysiology, University Hospital of La Princesa, Diego de León Street 62, \\ 28006 Madrid, Spain; lorenacarolina.vega@salud.madrid.org \\ 2 Department of Neurosurgery, University Hospital of La Princesa, Diego de León Street 62, \\ 28006 Madrid, Spain; cristinatorresdiaz@yahoo.es (C.V.T.); martasoti@yahoo.es (M.N.) \\ * Correspondence: jesus.pastor@salud.madrid.org; Tel.: +34-915-202-213
}

Received: 1 October 2019; Accepted: 3 November 2019; Published: 7 November 2019

\begin{abstract}
Deep brain stimulation (DBS) requires precise localization, which is especially difficult at the thalamus, and even more difficult in anesthetized patients. We aimed to characterize the neurophysiological properties of the ventral intermediate (V.im), ventral caudal (V.c), and centromedian parvo (Ce.pc) and the magnocellular (Ce.mc) thalamic nuclei. We obtained microelectrode recordings from five patients with refractory epilepsy under general anesthesia. Somatosensory evoked potentials recorded by microelectrodes were used to identify the V.c nucleus. Trajectories were reconstructed off-line to identify the nucleus recorded, and the amplitude of the action potential (AP) and the tonic (i.e., mean frequency, density, probability of interspike interval) and phasic (i.e., burst index, pause index, and pause ratio) properties of the pattern discharges were analyzed. The Mahalanobis metric was used to evaluate the similarity of the patterns. The mean AP amplitude was higher for the V.im nucleus $(172.7 \pm 7.6 \mu \mathrm{V})$ than for the other nuclei, and the mean frequency was lower for the Ce.pc nucleus $(7.2 \pm 0.8 \mathrm{~Hz})$ and higher for the V.c nucleus $(11.9 \pm 0.8 \mathrm{~Hz})$ than for the other nuclei. The phasic properties showed a bursting pattern for the V.c nucleus and a tonic pattern for the centromedian and V.im nuclei. The Mahalanobis distance was the shortest for the V.im/V.c and Ce.mp/Ce.pc pairs. Therefore, the different properties of the thalamic nuclei, even for patients under general anesthesia, can be used to positively define the recorded structure, improving the exactness of electrode placement in DBS.
\end{abstract}

Keywords: deep brain stimulation; epilepsy; extracellular action potentials; microelectrode recording

\section{Introduction}

The human thalamus is a complex egg-shaped structure composed of more than 50 different nuclear groups [1]. Some of these groups, such as the anterior (Ant) or centromedian (Ce) groups, have been used to treat epilepsy by means of deep brain stimulation (DBS) [2-7]. The Ant nucleus is anatomically well isolated and is usually addressed by neuronavigation and imaging techniques, without the help of microelectrode recording (MER) or other neurophysiological techniques $[8,9]$. However, the Ce is surrounded by different nuclei, including the ventral intermediate thalamic nucleus (V.im), ventral caudal thalamic nucleus (V.c.), or parafascicularis (Pf).

The first requirement for achieving a good outcome after DBS, optimizing the power provided by the batteries and decreasing secondary effects, is to ensure the appropriate nucleus is addressed. The human thalamus, except for some nuclei (e.g., the previously mentioned Ant nucleus or geniculate nucleus), has no anatomical landmarks that permit the selection of a definite nucleus within millimeters. Therefore, it is extremely important to have neurophysiological clues that allow us to identify during MER, in a positive way, the nucleus being recorded. These clues are obviously more relevant in 
anaesthetized patients, in which communication with the patient is not possible. In addition to MER, other physiological tests can be performed during DBS surgery to identify nuclei, such as the cellular response to voluntary or passive movements of members, tactile stimulus, or paraesthesia induced by electrical stimulation $[10,11]$. However, most of these responses (except response to tactile stimuli) are unspecific, and all of them need conscious collaboration with the patient, and therefore cannot be assessed in anaesthetized patients.

Recently, we showed [12] that the V.c. nucleus can be positively identified in anaesthetized patients by somatosensory evoked potentials (SSEP). Then, the nearby nuclei, namely, the V.im or Ce nucleus, can be identified during MER. Therefore, we can analyze neurophysiological properties and identify them even in anaesthetized patients.

For the reasons indicated above, DBS surgery is performed in awake patients to receive help from the patient during the activation maneuvers. This procedure is a fatiguing and stressful situation for the patient and for the surgical team, who are compelled to perform the surgery as fast as possible to avoid distress in the patient. Obviously, it would be preferable to do DBS in anaesthetized if we can ensure at least the same level of accuracy of anatomo-physiological localization in both anaesthetized and non-anaesthetized patients [13]. This method should be sufficiently robust to conserve some of the relevant physiological properties observed in awake patients during anesthesia. In other words, it must be invariant with respect to anesthesia.

The discharge properties of neurons depend on the set of ionic conductances (membrane excitability) and synaptic connections to other neural structures $[14,15]$. Both kinds of properties (excitability and connectivity) are highly specific for every neural structure. Therefore, we postulate that, although modified by anesthesia [16-18], some properties of connectivity and, in the strongest way, the properties of membrane excitability will be conserved and sufficiently different between several thalamic nuclei, allowing a positive identification of each nucleus.

The aim of our study was to characterize the neurophysiological properties of several thalamic nuclei, namely, the mean amplitude of the action potential (AP), mean frequency of the discharge, density of the neurons, and tonic and phasic properties of the discharge. Some authors have noted differences in the outcome between DBS stimulation of the centromedian magnocellular (Ce.mc) and centromedian parvocellular (Ce.pc.) nuclei [2,3]; therefore, we have analyzed both subdomains separately.

The preliminary results were published in an abstract form [19].

\section{Materials and Methods}

For this work, we followed the Schaltenbrand-Wahren (SW) atlas nomenclature [20]. Then, the structure referred to as the parafascicullaris/centromedian Pf/CM in the literature [21,22] is referred to as the $\mathrm{Ce}$, and the ventro-posterolateral and ventro-posteromedial (VPL/VML) nuclei are referred to as the V.c. [23,24].

\subsection{Patients and Surgery}

We studied 5 patients ( 3 men, 2 women, Table 1) diagnosed with generalized epilepsy undergoing surgery for chronic DBS treatment in the Ce. All patients freely provided informed consent to participate in the procedures approved by the Hospital La Princesa Ethics Board.

The patients were initially assessed for study eligibility using a presurgical evaluation in our center $[25,26]$, and patients undergoing resective surgery were excluded. Most of the patients were treated using vagus nerve stimulation (VNS), but after a two-year period of inadequate results, DBS in the Ce nucleus was proposed. During surgery, VNS was turned off and, immediately after recovery, the stimulations were continued at the same schedule. 
Table 1. Clinical features of the group studied.

\begin{tabular}{ccccccccc}
\hline Pat. & Sex & $\begin{array}{c}\text { Age } \\
\text { (years) }\end{array}$ & $\begin{array}{c}\text { History } \\
\text { (years) }\end{array}$ & Etiology & v-EEG & MRI Result & VNS & AED \\
\hline 1 & $\mathrm{~F}$ & 37 & 31 & Genetic $^{1}$ & GE & Normal & Yes & PGB, CBZ, CNZ \\
2 & $\mathrm{~F}$ & 18 & 12 & LGS & GE & Dysplasia LF & No & RUF, VPT \\
3 & $\mathrm{M}$ & 34 & 27 & Genetic $^{2}$ & EG/EE & Normal & Yes & VPT, PGB, LAC, ZNS \\
4 & $\mathrm{M}$ & 27 & 27 & LGS & GE & Normal & No & LVZ, OXC, LAC, CZM \\
5 & $\mathrm{M}$ & 30 & 23 & Structural $^{2}$ & GE/EE & Dysplasia biFT & Yes & TPM, VPT \\
\hline
\end{tabular}

AED: Anti-epileptic drugs. biFT: Bilateral fronto temporal. CBZ: Carbamacepin. CNZ: Clonacepam. CZM: Clobazam. F: Female. M: Male. EE: Epileptic encephalopathy. GE: Generalized epilepsy. LAC: Lacosamide. LF: Left frontal. LGS: Lennox-Gastaut syndrome. LTG: Lamotrigine. LVZ: Levetiracetam. OXC: Oxcarbamacepin. PGB: Pregabalin. RUF: Rufinamide. TPM: Topiramate. VPT: Valproate. ZNS: Zonisamide. ${ }^{1} 20$ ring-chromosome syndrome. ${ }^{2}$ Tuberous sclerosis.

All patients were operated on while under general anesthesia using propofol $(5.48 \pm 0.28 \mathrm{mg} / \mathrm{kg} / \mathrm{h}$, $[4.5,6.2])$ and remifentanil $(0.12 \pm 0.02 \mu \mathrm{g} / \mathrm{kg} / \mathrm{min},[0.1,0.2])$, maintaining a bispectral index (BIS) between 40 and 45 . Neuromuscular blocks were performed with cis-atracurium $(0.5 \mathrm{mg} / \mathrm{kg})$. No changes in anti-epileptic treatment was performed during the surgery.

The thalamus was identified using a $1.5 \mathrm{~T}$ magnetic resonance imaging (MRI) scanner (General Electric $($, Fairfield, CT, USA), and the coordinates were located stereotactically with a neuronavigator (BrainLab®, Feldkirchen, Germany). The coordinates were calculated by fusing the MRI images and CT scans, according to the SW atlas. For thalamic DBS electrode placement, a tentative initial target was selected in the Ce $(x=8, y=-10, z=0)$. All the coordinates corresponded to the mid-intercommissural AC-PC line (anterior commissure-posterior commissure). Neuronal recordings (Leadpoint $(\AA$, Minneapolis, USA) were obtained, starting at $10 \mathrm{~mm}$ above the target and progressing in increments of $0.5 \mathrm{~mm}$. Microelectrode recordings (MER- FHC@, Maine, USA) were obtained for both spontaneous and sensory-evoked activity until the inferior border of the thalamus was confirmed by the absence of neuronal activity. The impedance was always higher than $900 \mathrm{k} \Omega(1696 \pm 80 \mathrm{k} \Omega$, $(900,2900))$.

MERs were obtained through four microelectrodes placed $2 \mathrm{~mm}$ apart and placed (usually) at anterior, center, posterior, and lateral locations; in patient 5 only, the posterior electrodes were replaced by medial electrodes. Neuronal recordings (Leadpoint $₫$, Minneapolis, USA) of 15-90 s duration were obtained, beginning $10 \mathrm{~mm}$ above the target and progressing in steps of $0.5 \mathrm{~mm}$. MER were obtained from both spontaneous and sensory-evoked activity until the inferior border of the thalamus was confirmed by the absence of neuronal activity.

The Microdrive was fixed to a stereotactic Leksell Coordinate Frame (Elekta $囚$, Stockholm, Sweden). The bandwidth for spontaneous activity was $200 \mathrm{~Hz}-5 \mathrm{kHz}$, and the sample rate was $24 \mathrm{kHz}$; for the SSEPs only, the bandwidth was $2 \mathrm{~Hz}-5 \mathrm{kHz}$. A notch filter was not used. However, in two trajectories, the recordings were performed with the notch filter $(50 \mathrm{~Hz})$.

After the Ce was identified, a quadripolar DBS electrode was implanted. During the same surgery, the leads were connected to an implanted programmable stimulator (Kinetra, Medtronic®in three cases and Libra XP, Saint Jude®in two) placed in a pectoral or abdominal location.

\subsection{Somatosensory Evoked Potentials}

Somatosensory evoked potentials started at $8 \mathrm{~mm}$ above the theoretical target and repeated at $1 \mathrm{~mm}$ intervals until the end of the thalamus was reached. The lower border of the thalamus was easily identified by the absence of AP. Two rounds of 500 pulses were delivered at each point. The stimulation was elicited by electrical stimulation of the contralateral median nerve at the wrist at an intensity 1.5 times higher than the motor threshold (defined at the beginning of the surgery) with $200 \mu$ s pulses at a frequency of $7.1 \mathrm{~Hz}$ [19]. Responses at the cervical point (C2-Fpz) and both parietal regions (C3'/C4'-Fpz) were recorded by $18 \mathrm{~mm}$ subdermal needles (SGM®, Ljubiceva, Croatia) with a bandwidth of 10-1500 Hz (notch filter off). Simultaneously, the activity recorded through the four microelectrodes was averaged across the trials (Appendix A, Figure A1a). 
The presence of high frequency oscillations (HFO) of approximately $800 \mathrm{~Hz}$ was used as a specific landmark of the presence of the V.c nucleus (Appendix A, Figure A1a). A detailed analysis of these responses is beyond the scope of this manuscript (see [12]).

\subsection{Reconstruction of the Trajectory}

A detailed description of the reconstruction can be found in a previous study [12]. In brief, we consider coordinate $z=0$ the last recording inside the thalamus defined by the presence of an AP. The anteroposterior and lateral coordinates were obtained from the postoperative MRI scans performed one month after the surgery. Using this point and the stereo-tactic angles, we reconstructed the real trajectory of the electrode in a three-dimensional space in $1 \mathrm{~mm}$ intervals. Therefore, using the SW map, we were able to identify in which nucleus each electrode was located throughout a trajectory (Appendix A, Figure A2).

\subsection{Analysis of Discharge Properties}

We collectively analyzed the properties of discharge. To identify the neurons, we performed a sorting spike that, briefly, included the next steps. Data were exported to ASCII files, and analyses were performed off-line with bandwidth $0.5-5 \mathrm{kHz}$. Action potentials were identified and grouped according to these properties: Maximum and minimum amplitude, maximum and minimum value of the first derivative and duration of depolarizing and repolarizing phases. Using Mahalanobis metric, we clustered AP from the same neuron [12]. We can distinguish between tonic and phasic properties [24]. The following tonic properties were identified:

1. Amplitude of the AP, measured from peak to peak (in $\mu \mathrm{V}$ ). This property is not a tonic property, but is commonly used in clinical practice; therefore, we included it in this group.

2. Mean frequency and standard deviation of the raw trace and for every neuron. Both values were obtained from the inverse of the instant frequency.

3. Density, defined as the number of cells recorded by every electrode at one position. AP sorting was performed by clustering by using the Mahalanobis distances (see below) of several properties of the AP (e.g., amplitude and duration of positive and negative phases and maximum and minimum value of the first derivative). The maximum number of cells allowed to be identified by this mean was chosen as 5 .

4. Probability density functions (pdf) of the inter-spike interval (ISI) for every neuron. Relative frequency was computed for $1 \mathrm{~ms}$ bins, and the probability/bin $\left(p_{i}\right)$ was calculated with the following expression:

$$
p_{i}=\frac{f_{i}}{\sum_{j=1}^{N} f_{j}}
$$

where $f_{i}$ is the frequency for the $i$-bin and $N$ is the total number of bins.

To study the structure of these inter-spike interval histograms, we fitted the data to a double exponential decay function, using the following expression:

$$
y(x)=\alpha+\beta e^{-\gamma x}+\delta e^{-\varepsilon x}
$$

where $\alpha, \beta, \gamma, \delta$, and $\varepsilon$ are the constants to be fitted. The least square sum method was used to fit the empirical data to the defined functions.

The properties of phasic activity analyzed were as follows [27,28]:

1. Burst index (BI) is defined as the ratio between the number of ISI $<10 \mathrm{~ms}$ and the number of ISIs

$>10 \mathrm{~ms}$. It represents the number of bursts of discharges with respect to individual discharges, and is calculated as follows:

$$
B I=\frac{N_{I S I<10 m s}}{N_{I S I>10 m s}}
$$


2. Pause index (PI), defined as the ratio between the number of ISIs $>50 \mathrm{~ms}$ and the number of ISIs $<50 \mathrm{~ms}$, is calculated as follows:

$$
P I=\frac{N_{I S I>50 m s}}{N_{I S I<50 m s}}
$$

3. Pause ratio (PR), defined as the total duration of pauses (ISI $>50 \mathrm{~ms}$ ) divided by the total duration of no-pauses (ISI $<50 \mathrm{~ms}$ ). Although similar in name, the information obtained is different from that of the PI, and the PR is calculated as follows:

$$
P R=\frac{\sum_{i=1}^{N} I S I_{>50 \mathrm{~ms}}^{i}}{\sum_{j=1}^{N} I S I_{<50 \mathrm{~ms}}^{j}}
$$

Considering that the mean frequencies obtained were lower than those in awake patients, we computed all of these measurements using different pairs of threshold times instead of 10/50 ms. In fact, we attempted to use 20/100 and 50/400 ms.

All analyses were performed in custom MATLABß2018 scripts.

\subsection{Evaluation of Global Similarity}

Global comparisons between different nuclei cannot be performed adequately with pairs of variables. We wanted to ascertain the distance (e.g., dissimilarity) between all four nuclei considering all the variables. We measured every nucleus as a point in a 6-dimensional space, defined as follows:

$$
n u c=(\text { amplitude, frequency, density, BI, PI, PR }) ; n u c=\{C e . m c, \text { Ce.pc, V.im, V.c }\}
$$

Obviously, we cannot represent a 6-dimensional space in a two-dimensional figure. However, we can calculate the distance between every pair of nuclei. Therefore, the smaller the distance is between a pair, the more similarity there is between the nuclei. To that end, we used the Mahalanobis distance [29]. First, we computed the covariance matrix (S) with variances $\left(s_{i}^{2}\right)$ on the main diagonal. For every pair of nuclei $(i, j)$, we computed the distance $\left(d_{i j}\right)$ according to the following expression:

$$
d_{i j}=\left[\left(n u c_{i}-n u c_{j}\right) S^{-1}\left(n u c_{i}-n u c_{j}\right)^{\prime}\right]^{1 / 2}
$$

where $\left(n u c_{i}-n u c_{j}\right)^{\prime}$ is the transpose vector of $\left(n u c_{i}-n u c_{j}\right)$.

\subsection{Statistics}

Kurtosis $(K)$ was computed for every group, and only signals with values between 2 and 8 were considered signals from only one group [30]. Extreme outliers were removed progressively until $K<8$. Statistical analysis was applied only to these homogeneous groups.

Statistical comparisons between groups were performed using the z-score, Student's t-test, or ANOVA for data with normal distributions, and for data that failed the normality tests, the Mann-Whitney Rank sum test or Kruskal-Wallis analysis of variance were used. Normality was evaluated using the Kolmogorov-Smirnov test. SigmaStat $₫ 3.5$ software (Point Richmond, CA, USA) was used for statistical analysis. Instead of using rank as a dispersion measure, we used a 25-75 interquartile range, which is shown between brackets. In this context, a group refers to a set of measures of the same variable obtained from the same nucleus, e.g., mean amplitude AP, frequency, BI.

Pearson's correlation coefficient was used to study the linear dependence between the variables. Linear regression significance was evaluated by a contrast hypothesis against the null hypothesis $\rho=0$ using the formula:

$$
t=\frac{r \sqrt{N-2}}{\sqrt{1-r^{2}}}
$$


which describes a Student distribution with $N-2$ degrees of freedom.

The significance level was set at $p=0.05$. The results are shown as the mean $\pm \mathrm{SEM}$, except where otherwise indicated. As stated above, the $25-75$ interquartile range is reported between brackets, and the median is referred to as Med.

\section{Results}

The presence of high frequency oscillations (approximately $800 \mathrm{~Hz}$ ) and the reconstructed trajectories allowed us to identify the nuclei where raw recordings were coming up (Appendix A, Figures A1 and A2), assigning a cumulative trajectory (computed from all the tracks and from all the patients) of $171 \mathrm{~mm}$ to the V.c., $99 \mathrm{~mm}$ to the Ce, and $83 \mathrm{~mm}$ to the V.im. This method [12] has been proven to be robust in the identification of different nuclei and is extremely important for our purpose to avoid biases from the mixing of recordings from different nuclei.

The presence of a raw MER between 15 and $90 \mathrm{~s}$ at each point ensured that we had a significant number of extracellular APs. For every nucleus, we recorded a minimum of 1100 action potentials and a minimum of 41 traces. Examples of raw traces for the Ce, V.im, and V.c are shown in Appendix A, Figure A2.

\section{Tonic Properties}

These properties are commonly reported in the literature and are essentially the only properties used in the clinical assessment of thalamic nuclei, even though other mathematical tools have been used in previous reports $[27,28,31]$. However, neither the complete list of nuclei addressed in this manuscript, nor the level of detail analyzed, has been previously described in humans.

Usually, the amplitude of the AP is not considered for the clinical identification of nuclei in part because the variation in the amplitude is excessively high. However, we compared this property for the four thalamic nuclei addressed in this work. The mean AP amplitudes for Ce.mc, Ce.pc, and V.c were not different $(82.7 \pm 2.4, \mathrm{Med}=86.9,(75.4-95.2), 93.3 \pm 3.6, \mathrm{Med}=91.3,(88.1-103.6)$, and $133.4 \pm 3.8$, Med $=136.9,(124.4-142.3) \mu \mathrm{V}$, respectively), but the mean AP amplitude was higher for $\operatorname{V.im}(p<0.01)$ than for the other nuclei (Figure 1a), as it showed an amplitude nearly double that of the centromedian nuclei $(172.7 \pm 7.6, \mathrm{Med}=169.1,(156.6-191.6))$.

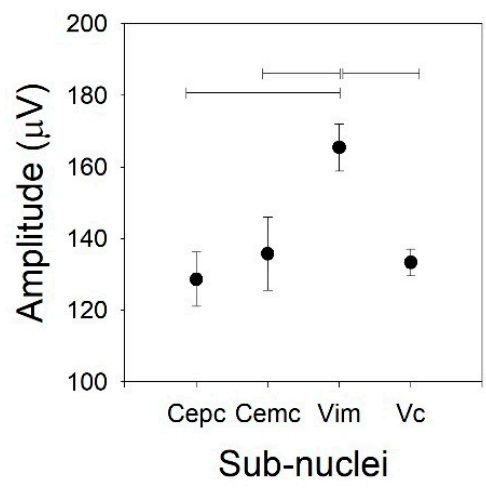

(a)

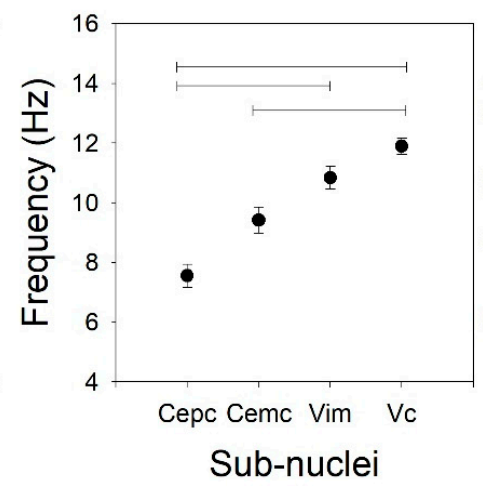

(b)

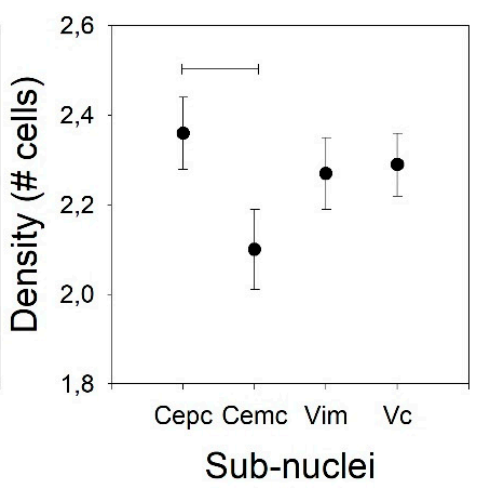

(c)

Figure 1. Tonic properties for the four nuclei. (a) Amplitude of the action potential (AP), (b) mean frequency of the raw traces, and (c) density of the cells. Horizontal lines indicate statistically significant differences between pairs of nuclei based on the Kruskal-Wallis analysis of variance test.

Not only the AP amplitude was different for the different nuclei, but the firing rate was also different (Figure 1b). In fact, there were low values for both centromedian subdomains $(7.2 \pm 0.8$, $\mathrm{Med}=10.5,(5.2-14.8)$ and $9.4 \pm 0.8, \mathrm{Med}=8.1,(5.0-11.4) \mathrm{Hz}$ for the Ce.pc and Ce.mc, respectively), which were not different. The frequency was high for the V.im $(10.3 \pm 0.6, \mathrm{Med}=10.8,(8.7-12.9) \mathrm{Hz}$, 
significantly different from the Ce.pc $-p<0.05$, but not from the Ce.mc) and was the highest for the V.c $(11.9 \pm 0.8, \mathrm{Med}=11.8,(9.8-13.7) \mathrm{Hz}-p<0.01)$, even though the frequency of the V.c was similar to that of the V.im and different from those of both centromedian nuclei $(p<0.001)$.

The density of the cells recorded in every nucleus (Figure 1c) was similar for the Ce.pc, V.im, and V.c $(2.36 \pm 0.08, \mathrm{Med}=2.1,(1.98-2.56), 2.27 \pm 0.08, \mathrm{Med}=2.22,(2.19-2.42)$, and $2.29 \pm 0.07, \mathrm{Med}=2.35$, (2.25-2.42) cells/trace, respectively), but it was lower for the Ce.mc $(2.10 \pm 0.09$, Med $=2.01,(1.89-2.34)$ cells/trace $-p<0.05)$.

The inter-spike intervals for the different nuclei were pooled and are shown in Figure 2.

(a)

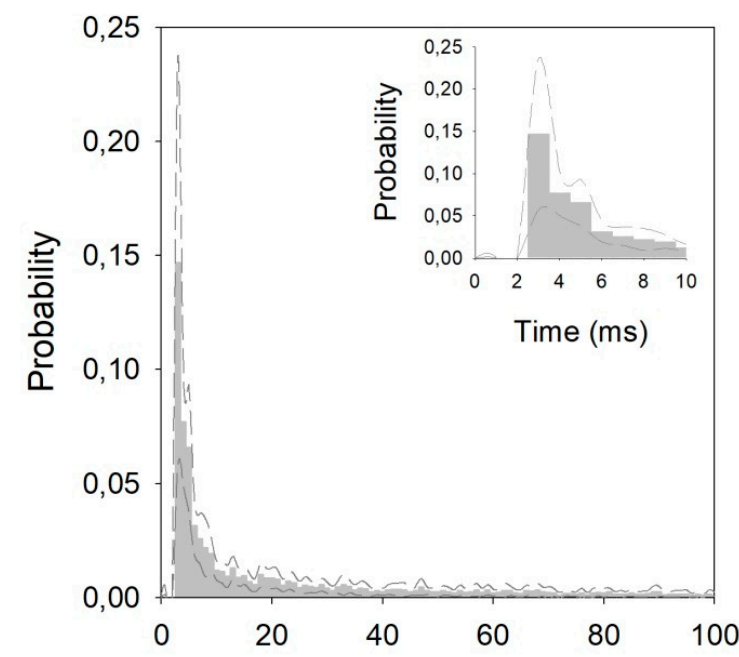

(b)

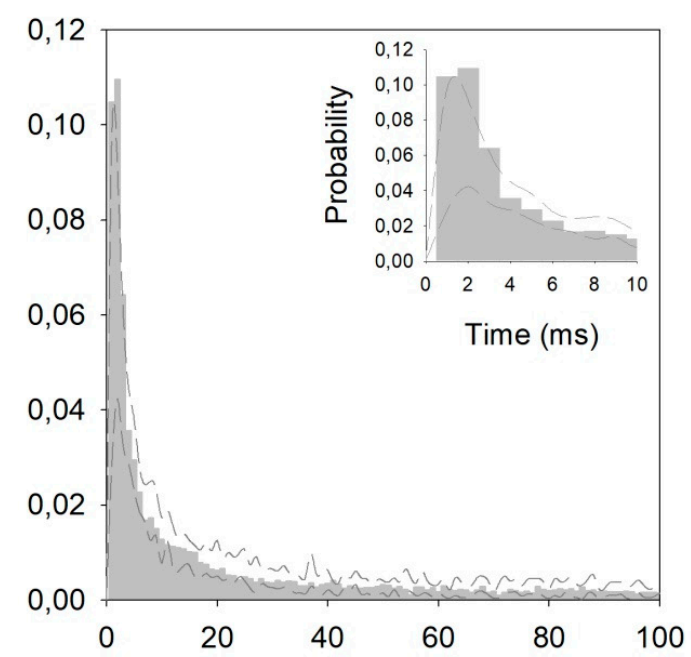

(c)

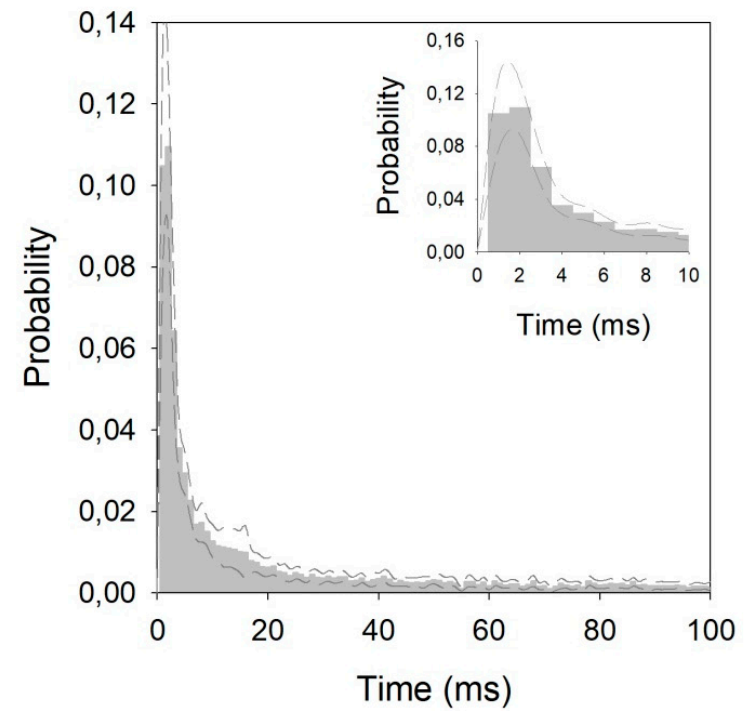

(d)

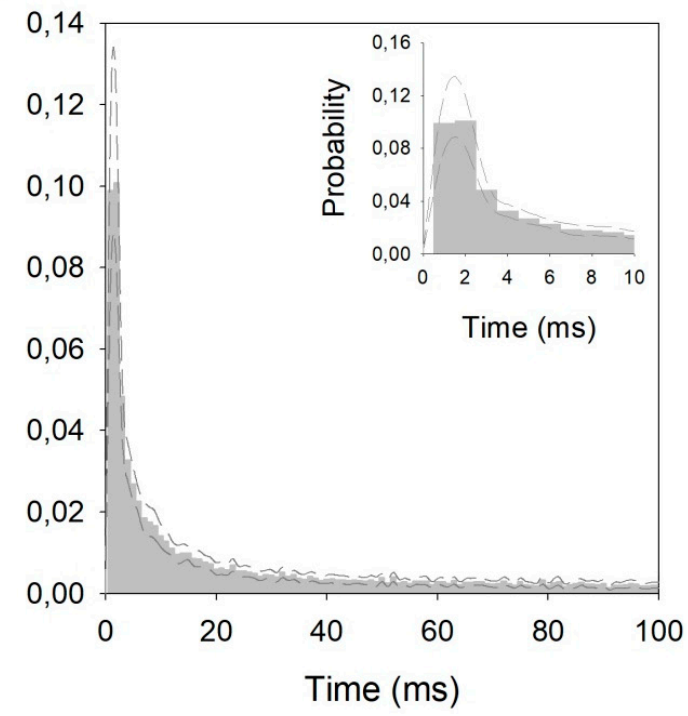

Figure 2. Probability density functions pooled for (a) centromedian magnocellular (Ce.mc), (b) centromedian parvocellular (Ce.pc) (c) ventral intermediate thalamic nucleus (V.im), and (d) ventral caudal thalamic nucleus (V.c). Discontinuous lines represent \pm 2.5 SEM. Insets show the details of the first $10 \mathrm{~ms}$. 
All the histograms were well fitted by double negative exponential functions, following Equation (2) (see Appendix B). It is important to observe that all the correlation coefficients are above 0.990 .

Finally, to characterize the phasic properties of the thalamic nuclei, we studied the BI, PI, and PR. All these properties are obtained from the raw pattern discharge, and are usually described by a binary plot (Figure 3) and inter-spike interval histograms. Periods between consecutive APs can be easily computed from binary plots, where each AP is represented by a vertical line.
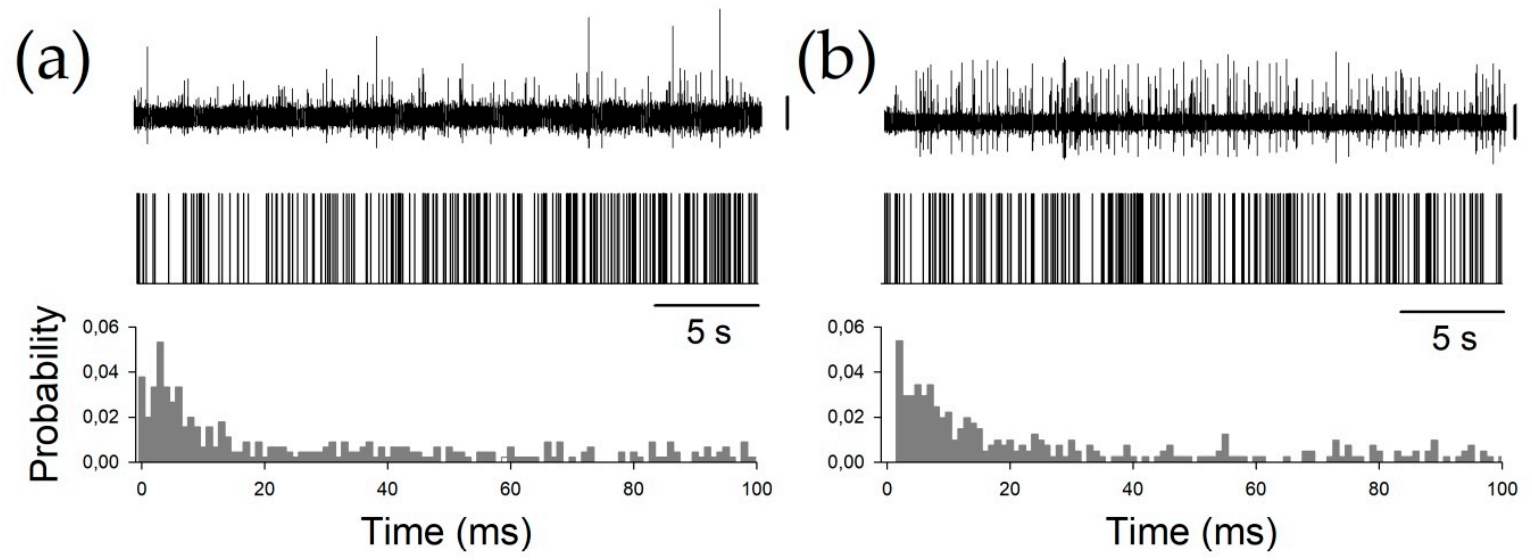

(c)
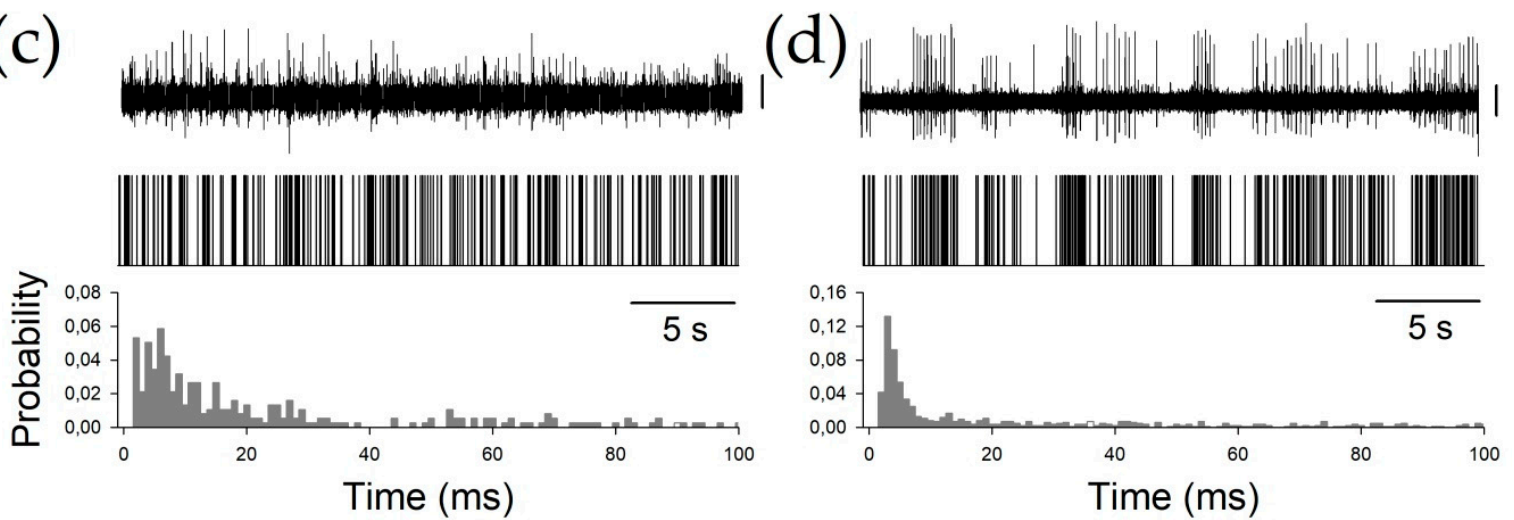

Figure 3. Examples of all the nuclei. (a) Ce.pc, (b) Ce.mc (c) V.c, and (d) V.im. For every panel, the upper row shows the raw trace obtained by the microelectrode recording (MER), the middle row shows the binary plots, and the bottom row shows the probability density functions (pdfs). Vertical calibration bars $=50 \mu \mathrm{V}$.

We used three different pairs of threshold times to compute the phasic variables. Therefore, we speculated that other pairs of times, different from 10/50 ms, could yield better results, taking into account that the mean frequencies obtained were lower than those published for awake patients. However, Figure 4 shows that the results are different for the BI and PI/PR. In fact, the discriminant capacity for BI is clearly lower for the $10 / 50 \mathrm{~ms}$ pair than for the other pairs. However, for PI and PR, the most discriminant pair threshold is $10 / 50 \mathrm{~ms}$.

In addition, the behavior of the BI for different pairs of thresholds is worse than that of the PI. In fact, a clear linearity can be observed for the three pairs of thresholds in the PI, but not for those in the BI. For the PR, only the 10/50 ms threshold pair shows linearity (Appendix C). Therefore, we used the 20/100 ms threshold pair for the remainder of the work. 


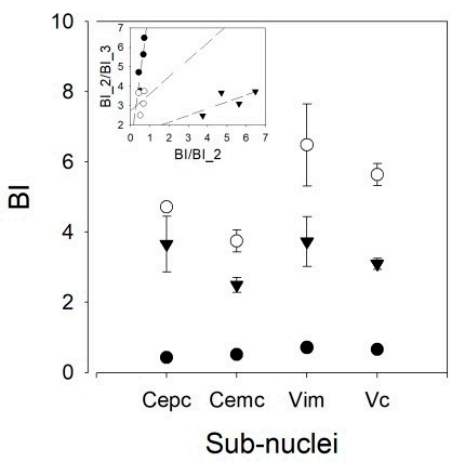

(a)

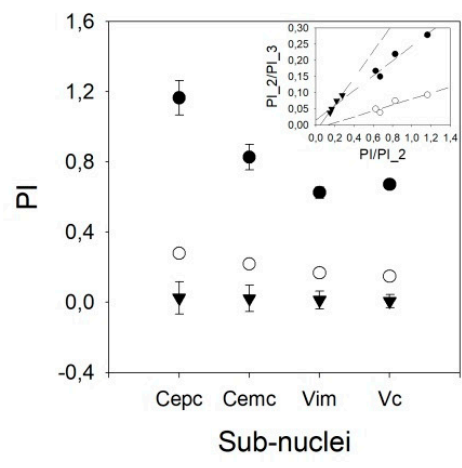

(b)

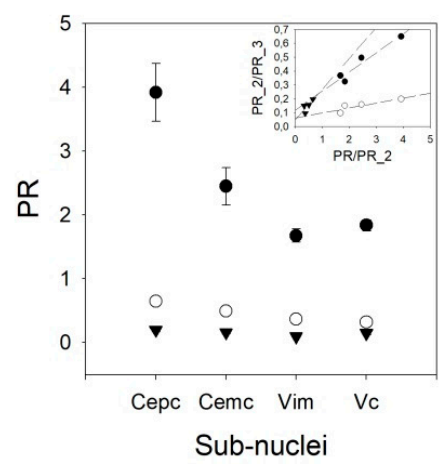

(c)

Figure 4. Graphs showing the values of (a) burst index (BI), (b) pause index (PI), and (c) pause ratio (PR) for all the nuclei for different pairs of times (solid dot $=10 / 50 \mathrm{~ms}$, empty dot $=20 / 100 \mathrm{~ms}$, and triangles $=50 / 200 \mathrm{~ms}$ ). Insets represent linear regressions for the values obtained for all the nuclei using different pairs of times (solid dots $=10 / 50$ vs. $20 / 100$, empty dots $=10 / 50$ vs. $50 / 200$ and triangles $=20 / 100$ vs. $50 / 200)$.

As shown in Figure 5, the BI for the V.im nucleus $(6.479 \pm 1.165, \mathrm{Med}=4.911,(3.008-5.866))$ is higher than those for the Ce.pc and Ce.mc $(4.716 \pm 2.103$, Med $=2.465$, (1.799-3.125) and 3.744 \pm 0.317 , Med $=3.749$, (2.521-4.927), $p<0.05$ and $p<0.01$, respectively) but is not different from that of the V.c $(5.633 \pm 0.317, \mathrm{Med}=4.964,(3.533-7.096))$. Nonetheless, the BI for the V.c is also higher than that of the Ce.pc $(p<0.05)$. The PI and PR were higher for the Ce.pc $(0.278 \pm 0.025$, Med $=0.26$, (0.169-0.368), 0.648 $\pm 0.065, \mathrm{Med}=0.586,[0.366-0.867]$ respectively for PI and PR) than for the V.im $(0.167 \pm 0.012, \mathrm{Med}=0.154,(0.120-0.226)$ and $0.367 \pm 0.029, \mathrm{Med}=0.331,(0.253-0.501), p<0.01)$ and the V.c $(0.149 \pm 0.068, \mathrm{Med}=0.140,(0.104-0.195)$, and $0.324 \pm 0.016, \mathrm{Med}=0.298,(0.218-0.427), p<0.001)$, but was not higher than that for the Ce.mc $(0.219 \pm 0.022, \mathrm{Med}=0.195,(0.153-0.258)$ and $0.496 \pm 0.057$, Med $=0.429,(0.327-0.581))$.

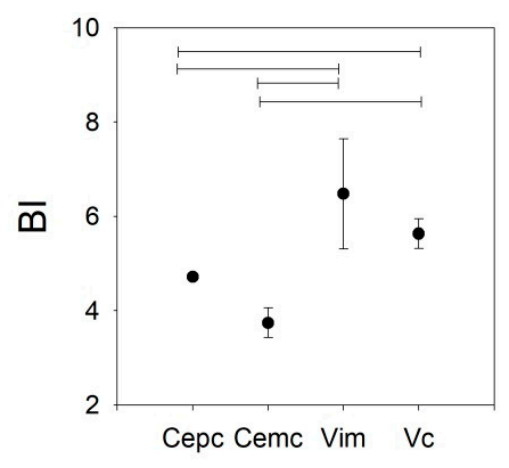

Sub-nuclei

(a)

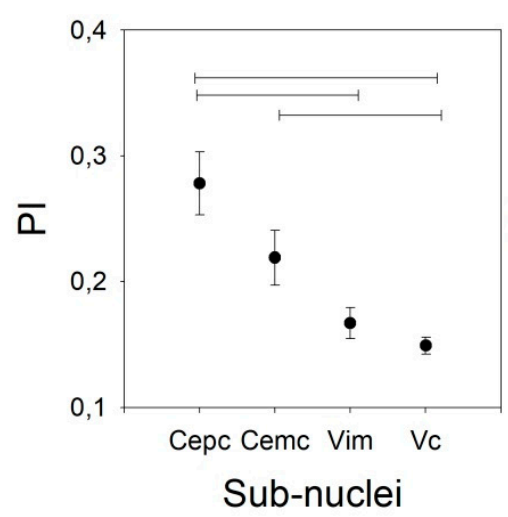

(b)

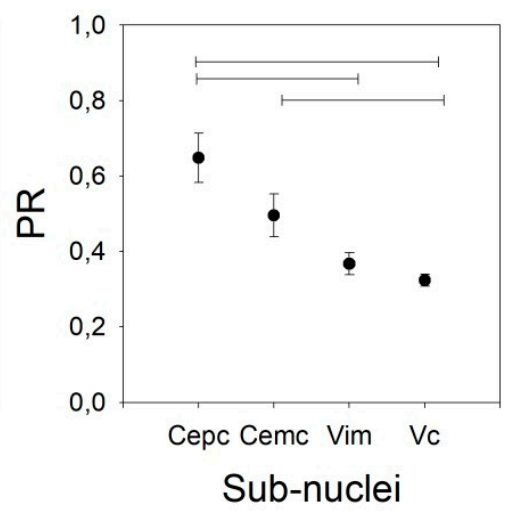

(c)

Figure 5. Phasic properties for the four nuclei for the 20/100 ms threshold pair. (a) BI, (b) PI, (c) PR. Horizontal lines indicate statistically significant differences between pairs of nuclei according to the Kruskal-Wallis analysis of variance test.

Finally, we addressed the similarity/dissimilarity between the global properties of the nuclei by using the Mahalanobis distance $(d)$ according to Equation (6). The sorted relations between pairs of nuclei are V.c-V.im $(d=2.178)<$ Ce.pc-Ce.mc $(d=3.086)<$ Ce.mc-V.c $(d=3.1857)<$ Ce.mc-V.im $(d=3.617)<$ Ce.pc-V.c $(d=4.038)<$ Ce.pc-V.im $(d=4.266)$. Therefore, the two nuclei that have a shorter distance (e.g., the two nuclei that are more similar) are V.im and V.c, followed by Ce.pc and Ce.mc, which are clearly separated from V.c and V.im. 


\section{Discussion}

We have shown in this work that the global properties of different thalamic nuclei in anaesthetized patients are different and can be used to identify the nuclei. The centromedian nucleus can be easily identified during MERs and distinguished from the other thalamic nuclei. These facts are relevant from the clinical point of view because this set of properties can be used to identify every nucleus with precision by MERs during DBS surgery.

Currently, the mean frequency discharge and the expertise of the neurophysiologist in identifying a raw record as phasic or tonic are the main tools used during MERs to identify the structure recorded. In awake patients, some other clues can be used, such as the response to active or passive limb movements, the presence of paresthesia and a neural response to light tactile stimulation $[10,11]$. Obviously, all these physiological activations cannot be used in anaesthetized patients. Moreover, these responses are unspecific to a considerable degree (except for the neural response to light touch), and therefore, a positive identification of diverse nuclei in awake patients is of great importance.

From a methodological viewpoint, it is important to consider that all patients were under general anesthesia, and this condition can modify neuronal discharge features and perhaps the synaptic functions of some thalamic nuclei [32], but the exact degree of the effect remains to be elucidated. However, it is known that the mean frequency discharge (a useful variable during MERs) [16-18,33] and other electrophysiological properties, such as local field potentials [34], are affected by anesthesia. However, there is evidence in a study of DBS of the subthalamic nucleus (STN) that localization with MER and patient comfort can be achieved with equal effectiveness in patients under generalized anesthesia compared with that in awake patients [35].

Another important consideration is that we have used a reconstruction of the real trajectory [12] to identify the placement for every electrode that can be plotted clearly in the SW planes. This approach can only be performed off-line because we need to identify the lower border of the thalamus. However, this approach has been shown to be sufficiently robust to identify nuclei, enabling raw traces from different patients pertaining to the same neural structure to be grouped.

The AP amplitude was different only for the V.im nucleus; the rest of the nuclei were analyzed, but parts of centromedian and V.c could not be distinguished. Extracellular recordings retain some of the properties of APs acquired intracellularly $[15,36,37]$, but amplitudes of extracellularly recorded APs do not depend solely on features of membrane excitability. In fact, the amplitudes of extracellular APs decrease rapidly as a function of the distance between the tip of the recording electrode and the soma [37]. However, the relative position between the electrode and soma is not the important factor. In addition, the local field potential depending on the contribution of the global sources of the environment can modify the amplitude [36]. The duration of an AP is a more robust measure that is not affected by any of these variables; therefore, we postulate that measurements of duration and other properties of an AP (number of phases, durations of them) can be more useful in identifying nuclei. This idea remains to be elucidated by future studies.

Obviously, the number of ISIs lower/greater than a defined value (e.g., 10, $50 \mathrm{~ms}$ ) depends on the pdf, which is related (not in a linear way, of course) to the mean frequency. In awake patients, the mean frequency described is higher than that in the patients in our study; therefore, the number of ISIs $<10 \mathrm{~ms}$ should be greater than that in the patients in our study $[17,18,28]$. We have shown that for lower frequencies, such as those obtained in anaesthetized patients, the 20/100 ms pair of threshold times is preferable to the pair of lower threshold times, $10 / 50 \mathrm{~ms}$, used in awake patients [28]. 
We have characterized every nucleus by a point in a 6th dimensional configuration space. This approach is typical of multivariate analysis in which every dimension represents different magnitudes and physical units. Therefore, every point describes the state of the system. It's quite useful to assess how close or far away two systems are, i.e., two points of this space; in other words, we need to describe a metric [38]. The Mahalanobis distance is a useful multivariate analysis tool used to measure the distance between a pair of points in a multidimensional space. It is based on Minkowski's distances, but in contrast to these, it does not depend on the existence of natural units of measure [39,40]. As we can observe from Equation (7), this metric is dimensionless and therefore, there are not inconsistencies in its use. We have shown that the similarity is high between the V.c and V.im, and is surprisingly low between both parts of the centromedian. The distance is large between the centromedian and V.im and even larger between the centromedian and V.c. The V.im nucleus has a high AP amplitude and a mean frequency between those of the centromedian and V.c, but some properties (e.g., PI and PR) are similar to those of the V.c.

Properties related to pattern discharge are scarcely used in clinical practice, but they have been analyzed for different structures addressed during DBS, such as the STN [41,42], Ant thalamic nuclei [43], and globus pallidus [21,44]. However, these properties have not been described for the nuclei analyzed in this work. We have shown here that even under general anesthesia, some properties of raw discharge are different for these thalamic nuclei. This is a very important fact, irrespective of whether these properties share a similar relationship in awake patients, because they can be used to provide a positive identification of thalamic structures only by MER without the need for conscious patient collaboration. However, other properties, as mean pdf (Figure 2), are not able to discriminate between nuclei. We cannot exclude a bias derived either from the small number of patients or an anesthetic effect. We need a bigger number of patients, and comparison with pdf obtained from awake patients to explain this fact. In a complex structure such as the thalamus, the placement of electrodes a couple of millimeters apart can involve a completely different nucleus. Hence, identifying the position at which the electrode is placed to the most accurate extent possible is extremely important. During DBS surgery, drainage of cerebro-spinal fluid or air entering inside the cranium can displace the target as much as several millimeters from its theoretical coordinates $[45,46]$. Identification of a neural structure by its electrophysiological properties prevents errors from occurring due to this morphological change of the brain.

In summary, it is important to understand that our data were obtained from a small number of patients. Although these data seem to be robust, we need a larger cohort to unequivocally establish these results.

\section{Conclusions}

In this work, we demonstrated that properties related to raw traces are different for different thalamic nuclei in anesthetized patients. Isolated single features (i.e., amplitude or frequency) cannot sufficiently discriminate between nuclei to identify a raw trace. However, it may be relatively easy to compute the properties described in this paper online to identify the structure from which the discharge is obtained. We have characterized the electrophysiological properties of the centromedian nucleus, which have not been previously described. We propose that this method can increase the accuracy of electrode placement in DBS and decrease the secondary effects and energy consumption derived from the incorrect placement of electrodes. 
Author Contributions: All authors (C.V.T., M.N., L.V.-Z. and J.P.) participated in data collection. J.P. and L.V.-Z. participated also in analysis and interpretation, and J.P. was responsible idea and concept of manuscript and its preparation. C.V.T., M.N., L.V.-Z. and J.P. have approved the submitted version of this manuscript.

Funding: This work was financed by a grant from the Ministerio de Sanidad FIS PI17/02193 and was partially supported by FEDER (Fonds Europeen de Developpement Economique et Regional).

Acknowledgments: The authors want to acknowledge the collaboration during surgeries by anaesthesiologists María Luisa Meilán and Eva de Dios.

Conflicts of Interest: The authors declare no conflict of interest. The funders had no role in the design of the study; in the collection, analyses, or interpretation of data; in the writing of the manuscript, or in the decision to publish the results.

\section{Appendix A}

Reconstructed trajectories and SSEP allowed us to identify the nucleus from which the recordings originated. In the first figure, we show examples of a raw recording and SSEP. The reconstruction of a trajectory for the four microelectrodes is also shown.

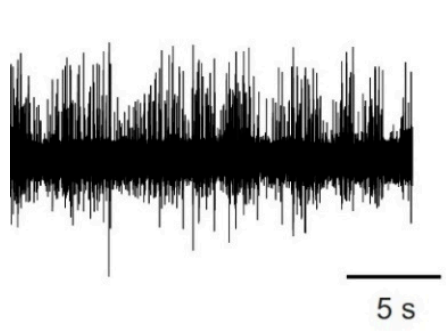

(a)

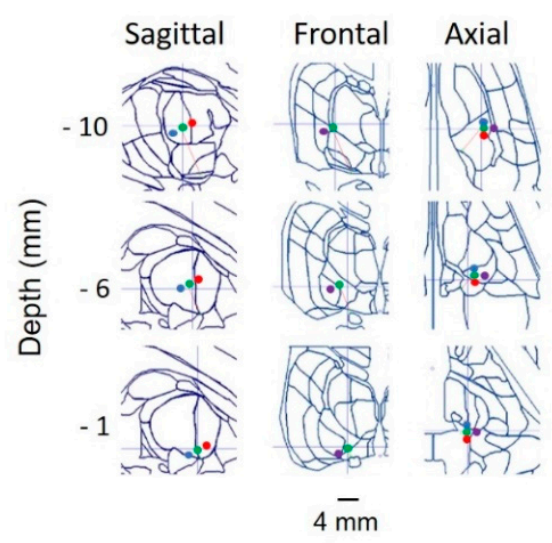

(b)

Figure A1. Identification of thalamic nuclei. (a) Raw MER (left) and high frequency oscillations (HFO) obtained by SSEP (right); (b) reconstruction of the trajectory from one patient, showing microelectrode (anterior $=$ blue, central $=$ green, posterior $=$ red, and lateral $=$ purple) positions at different depths superimposed onto an SW map. The numbers on the left indicate the true distance to the target.

In Figure A2, we show the raw records from all the nuclei studied and the placement of the electrode superimposed onto the SW atlas.

\section{Appendix B}

Probability density functions fit extremely well to negative double-exponential functions. In Figure A3, we plotted both exponential parts of the functions for each nucleus (removing the constant $\alpha$ ), and the values of the constants fitted are shown in Table A1. As we can observe, there are clearly two parts for every histogram; the first part between the peak, at 2-3 ms inter-spike interval, and another part 4-6 ms later. In this phase, the $\gamma$ constants for Ce.mc and V.c are very similar and are clearly slower than those for Ce.pc and V.im (which is the faster constant). The second phase is quite similar for all nuclei, especially for the Ce.mc and V.im, showing practically similar functions. Globally, these results show that the pdfs are quite similar, and the main differences between the nuclei are in the first $10 \mathrm{~ms}$. 


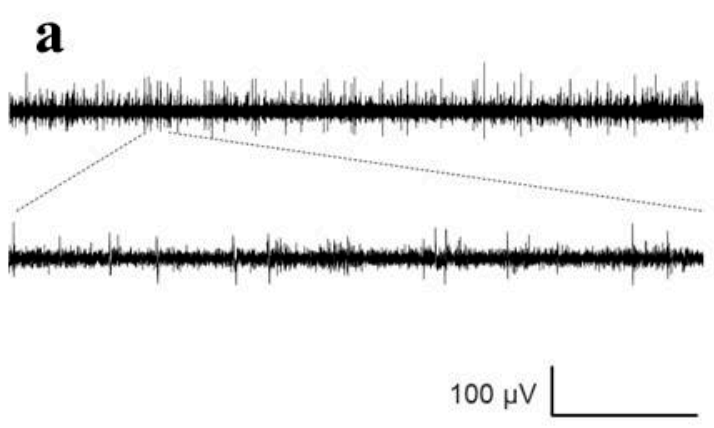

b
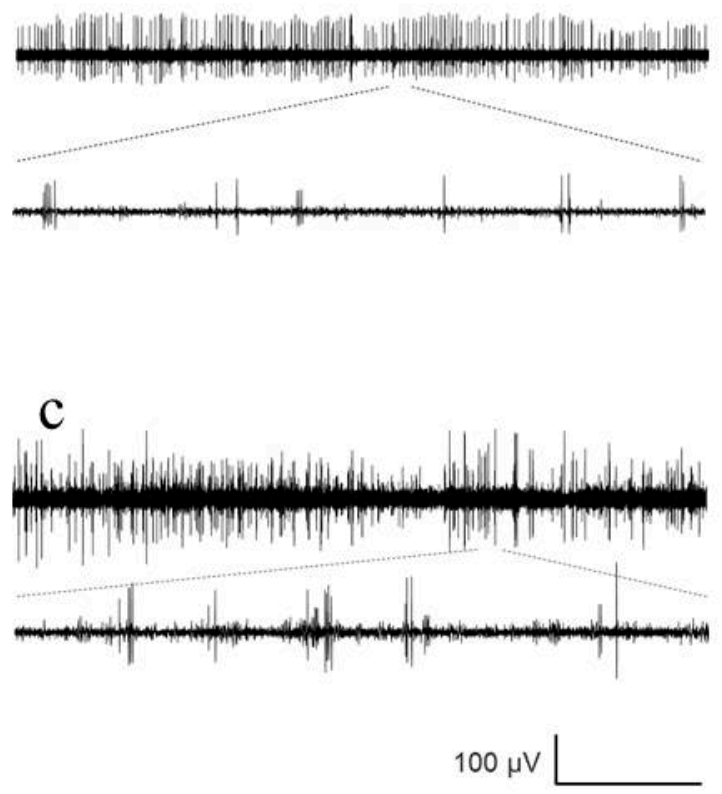

d

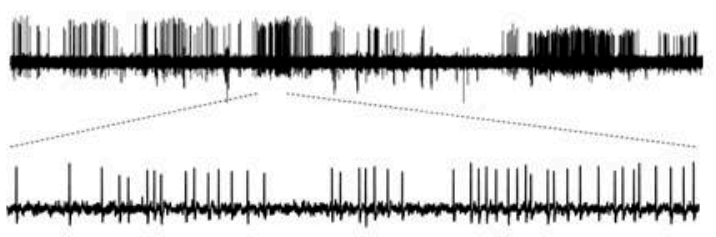

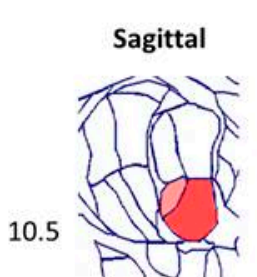
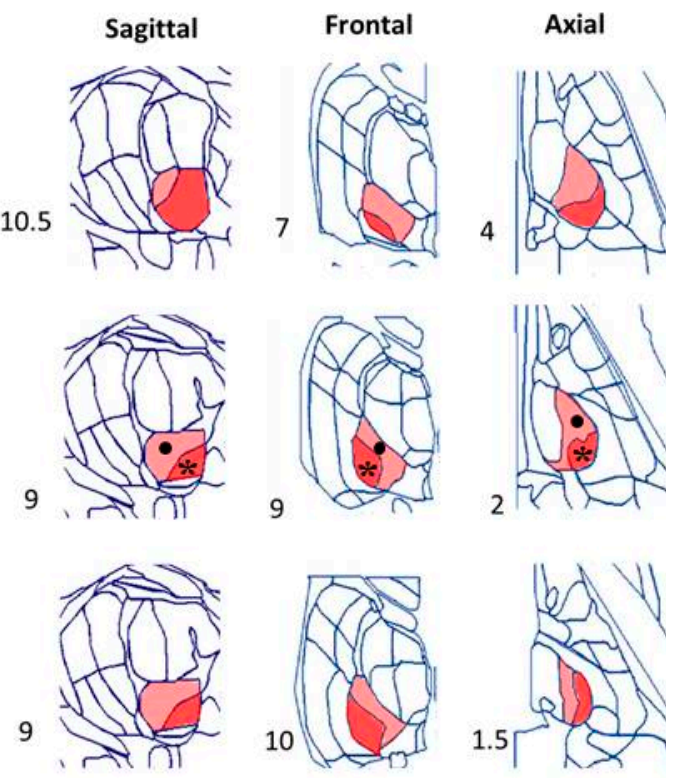

10.5
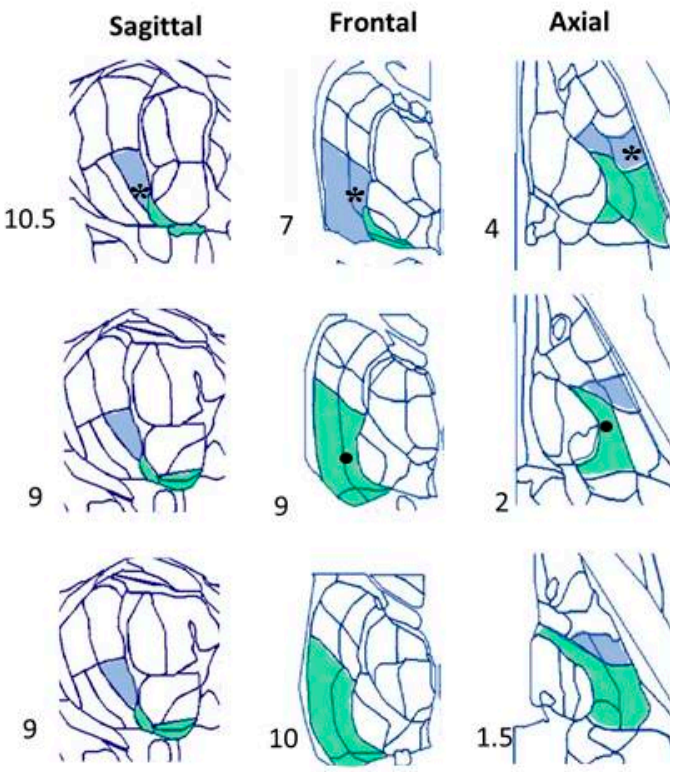

Figure A2. Recordings from different nuclei and positions identified by reconstruction. Raw traces from MER are in the left column, and different planes from the Schaltenbrand-Wahren (SW) atlas are in the right column. Horizontal calibration bar: $5 \mathrm{~s}$ for the upper traces and $167 \mathrm{~ms}$ for the lower traces. (a) Ce.mc (pale red), asterisk indicates the location of the recording, (b) Ce.pc. (strong red), the dot shows the location of the recording, (c) V.im (blue), asterisk indicates the location of the recording, and (d) V.c (green), indicated by a dot. Numbers show the distance for every axis from the mid-intercommissural AC-PC line. 


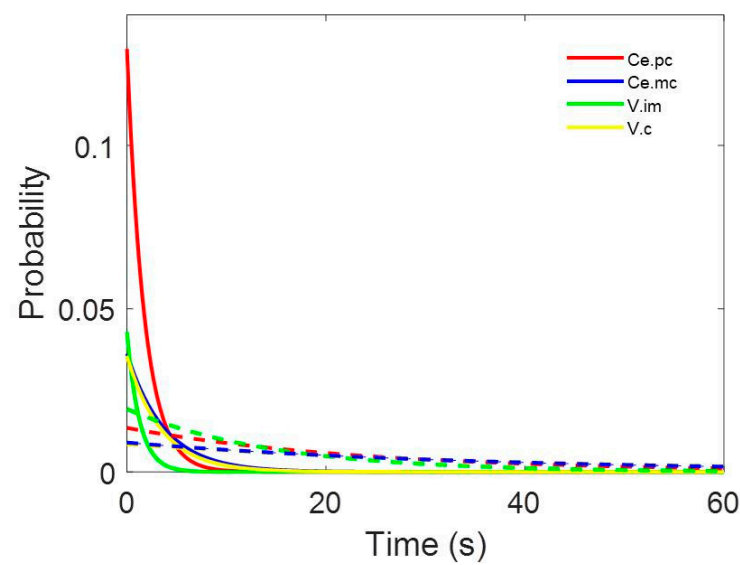

Figure A3. Representation of both parts of double exponential functions for Ce.pc (red), Ce.mc (blue), V.im (green), and V.c (yellow). Continuous lines represent the first part of equations $\left(\beta e^{-\gamma x}\right)$ and discontinuous ones the second parts $\left(\delta e^{-\varepsilon x}\right)$ of Equation (2).

Table A1. Parameters for the double negative exponentials functions fitted for pdf.

\begin{tabular}{ccccccc}
\hline Nucleus & $\boldsymbol{r}$ & $\boldsymbol{\alpha}$ & $\boldsymbol{\beta}$ & $\boldsymbol{\gamma}$ & $\boldsymbol{\delta}$ & $\boldsymbol{\varepsilon}$ \\
\hline Ce.pc & 0.9939 & 0.001 & 0.1296 & 0.5683 & 0.0135 & 0.0421 \\
Ce.mc & 0.9904 & 0.001 & 0.0363 & 0.2660 & 0.0090 & 0.0285 \\
V.im & 0.9929 & 0.0013 & 0.0429 & 0.7841 & 0.0193 & 0.0692 \\
V.c & 0.9925 & 0.001 & 0.0356 & 0.2899 & 0.0085 & 0.0262 \\
\hline
\end{tabular}

\section{Appendix C}

Linearity of phasic variables for different pairs of threshold times.

Table A2. Parameters for the double negative exponential functions fitted for pdf.

\begin{tabular}{cccccc}
\hline Index & Pair of Thresholds (ms) & Linear Function $[\mathbf{y}(\mathbf{x})]$ & $\boldsymbol{r}^{2}$ & $\boldsymbol{t}$ & $\boldsymbol{p}$ \\
\hline \multirow{3}{*}{ BI } & $10 / 50$ & $7.35 x+0.89$ & 0.675 & 2.038 & n.s \\
& $20 / 100$ & $0.87 x+2.74$ & 0.040 & 0.289 & n.s \\
& $50 / 200$ & $0.35 x+1.44$ & 0.513 & 1.451 & n.s \\
PI & $10 / 50$ & $0.23 x+0.01$ & 0.932 & 5.215 & $<0.01$ \\
& $20 / 100$ & $0.09 x+0.01$ & 0.849 & 3.347 & $<0.05$ \\
& $50 / 200$ & $0.41 x+0.02$ & 0.980 & 9.900 & $<0.001$ \\
PR & $10 / 50$ & $1.38 x+0.12$ & 0.933 & 5.261 & $<0.01$ \\
& $20 / 100$ & $0.04 x+0.06$ & 0.757 & 2.496 & n.s \\
& $50 / 200$ & $0.22 x+0.05$ & 0.602 & 1.739 & n.s \\
\hline
\end{tabular}

n.s = not significant. $\mathrm{BI}=$ burst index. $\mathrm{PI}=$ pause index. $\mathrm{PR}=$ pause ratio.

\section{References}

1. Nieuwenhuys, R.; Voogd, J.; van Huijzen, C. (Eds.) Diencephalo: Dorsal Thalamus. In The Human Central Nervous System; Springer: Berlin/Heidelberg, Germany, 2008; pp. 253-279.

2. Velasco, A.L.; Velasco, F.; Jiménez, F.; Velasco, M.; Castro, G.; Carrillo-Ruiz, J.D.; Fanghänel, G.; Boleaga, B. Neuromodulation of the centromedian thalamic nuclei in the treatment of generalized seizures and the improvement of the quality of life in patients with Lennox-Gastaut syndrome. Epilepsia 2006, 47, 1203-1212. [CrossRef]

3. Velasco, F.; Velasco, A.L.; Velasco, M.; Jiménez, F.; Carrillo-Ruiz, J.D.; Castro, G. Deep brain stimulation for treatment of the epilepsies: The centromedian thalamic target. Acta Neurochir. Suppl. 2007, 97, 337-342.

4. Valentín, A.; García-Navarrete, E.; Chelvarajah, R.; Torres, C.; Navas, M.; Vico, L.; Torres, N.; Pastor, J.; Selway, R.; Sola, R.G.; et al. Deep brain stimulation of the centromedian thalamic nucleus for the treatment of generalised and frontal epilepsies. Epilepsia 2013, 54, 1823-1833. [CrossRef] 
5. Torres, C.; Pastor, J.; Navarrete, E.G.; Sola, R.G. Estimulación cerebral profunda talámica para la epilepsia resistente. Rev. Neurol. 2011, 53, 99-106. [CrossRef] [PubMed]

6. Kim, S.H.; Lim, S.C.; Kim, J.; Son, B.C.; Lee, K.J.; Shon, Y.M. Long-term follow-up of anterior thalamic deep brain stimulation in epilepsy: A 11-year, single center experience. Seizure 2017, 52, 154-161. [CrossRef] [PubMed]

7. Yan, H.; Toyota, E.; Anderson, M.; Abel, T.J.; Donner, E.; Kalia, S.K.; Drake, J.; Rutka, J.T.; Ibrahim, G.M. A systematic review of deep brain stimulation for the treatment of drug-resistant epilepsy in childhood. J. Neurosurg. Pediatr. 2018, 23, 274-284. [CrossRef] [PubMed]

8. Herrman, H.; Egge, A.; Konglund, A.E.; Ramm-Pettersen, J.; Dietrichs, E.; Taubøll, E. Anterior thalamic deep brain stimulation in refractory epilepsy: A randomized, double-blinded study. Acta Neurol. Scand. 2019, 139, 294-304. [CrossRef] [PubMed]

9. Koeppen, J.A.; Nahravani, F.; Kramer, M.; Voges, B.; House, P.M.; Gulberti, A.; Moll, C.K.E.; Westphal, M.; Hamel, W. Electrical Stimulation of the Anterior Thalamus for Epilepsy: Clinical Outcome and Analysis of Efficient Target. Neuromodulation 2019, 22, 465-471. [CrossRef] [PubMed]

10. Obwegeser, A.A.; Uitti, R.J.; Turk, M.F.; Strongosky, A.J.; Wharen, R.E. Thalamic stimulation for the treatment of midline tremors in essential tremor patients. Neurology 2000, 54, 2342-2344. [CrossRef]

11. Wu, D.; Wang, S.; Stein, J.F.; Aziz, T.Z.; Green, A.L. Reciprocal interactions between the human thalamus and periaqueductal gray may be important for pain perception. Exp. Brain Res. 2014, 232, 527-534. [CrossRef]

12. Pastor, J.; Vega-Zelaya, L. A new potential specifically marks the sensory thalamus in anaesthetized patients. Clin. Neurophysiol. 2019, 130, 1926-1936. [CrossRef] [PubMed]

13. Vega-Zelaya, L.; Sola, R.G.; Pulido, P.; Pastor, J. Do we need to awake patients up during cortical surgery? J. Cancer Res. Updates 2018, 7, 84-96. [CrossRef]

14. Koester, J.; Siegelbaum, S.A. Membrane Potential. In Principles of Neural Science; Kandel, E.R., Schwartz, J.H., Jessell, T.M., Eds.; Elsevier: New York, NY, USA, 2000; pp. 125-139.

15. Gold, C.; Henze, D.A.; Koch, C.; Buzsáki, G. On the origin of the extracellular action potential waveform: A modeling study. J. Neurophysiol. 2006, 95, 3113-3128. [CrossRef] [PubMed]

16. Sloan, T.B. Anesthesia and motor evoked potential monitoring. In Neurophysiology in Neurosurgery; Deletis, V., Shils, J.H., Eds.; Academic Press: Amsterdam, The Netherlands, 2002; pp. 451-474.

17. Voss, L.J.; Sleigh, J.W.; Barnard, J.P.; Kirsch, H.E. The howling cortex: Seizures and general anesthetic drugs. Anesth. Analg. 2008, 107, 1689-1703. [CrossRef]

18. Hutchison, W.D. Electrophysiological findings in GPe and GPi. In Microelectrode Recording in Movement Disorder Surgery; Zvi, I., Kim, J.B., Eds.; Thieme: New York, NY, USA, 2004.

19. Vega-Zelaya, L.; Torres, C.; Sola, R.G.; Pastor, J. Characterization of thalamic nuclei and somatosensory evoked potentials in anesthetized humans. Clin. Neurophysiol. 2016, 127, 203-205. [CrossRef]

20. Hassler, R. Anatomy of the thalamus. In Introduction to Stereotaxis with an Atlas of the Human Brain; Schaltenbrand, G., Bailey, P., Eds.; Thieme: Stuttgart, Germany, 1959; pp. 230-290.

21. Peppe, A.; Gasbarra, A.; Stefani, A.; Chiavalon, C.; Pierantozzi, M.; Fermi, E.; Stanzione, P.; Caltagirone, C.; Mazzone, P. Deep brain stimulation of CM/PF of thalamus could be the new elective target for tremor in advanced Parkinson's Disease? Parkinsonism Relat. Disord. 2008, 14, 501-504. [CrossRef] [PubMed]

22. Semenova, U.; Raeva, S.; Sedov, A. Participation of the thalamic CM-Pf complex in movement performance in patients with dystonia. Mov. Disord. 2016, 31, 1398-1404. [CrossRef] [PubMed]

23. Owen, S.L.; Green, A.L.; Stein, J.F.; Aziz, T.Z. Deep brain stimulation for the alleviation of post-stroke neuropathic pain. Pain 2006, 120, 202-206. [CrossRef] [PubMed]

24. Nguyen, J.P.; Nizard, J.; Keravel, Y.; Lefaucheur, J.P. Invasive brain stimulation for the treatment of neuropathic pain. Nat. Rev. Neurol. 2011, 7, 699-709. [CrossRef] [PubMed]

25. Pastor, J.; Hernando-Requejo, V.; Domínguez-Gadea, L.; de Llano, I.; Meilán-Paz, M.L.; Martínez-Chacón, J.L.; Sola, R.G. Impact of experience on improving the surgical outcome in temporal lobe epilepsy. Rev. Neurol. 2005, 41, 709-716. [PubMed]

26. Sola, R.G.; Hernando-Requejo, V.; Pastor, J.; García-Navarrete, E.; DeFelipe, J.; Alijarde, M.T.; Sánchez, A.; Domínguez-Gadea, L.; Martín-Plasencia, P.; Maestú, F.; et al. Pharmacoresistant temporal-lobe epilepsy. Exploration with foramen ovale electrodes and surgical outcomes. Rev. Neurol. 2005, 41, 4-16. [PubMed]

27. Javre, J.; Baumann, T. Signal processing and pattern recognition. In Microelectrode Recording in Movement Disorder Surgery; Zvi, I., Kim, J.B., Eds.; Thieme: New York, NY, USA, 2004. 
28. Favre, J.; Taha, J.M.; Baumann, T.; Burchiel, K.J. Computer analysis of the tonic, phasic, and kinesthetic activity of pallidal discharges in Parkinson patients. Surg. Neurol. 1999, 51, 665-672. [CrossRef]

29. Mahalanobis, P.C. On the generalized distance in statistics. Proc. Natl. Inst. Sci. India 1936, 2, 49-55.

30. Peña, D.; Prieto, F.J. The kurtosis coefficient and the linear discriminant function. Stat. Probab. Lett. 2000, 49, 257-261. [CrossRef]

31. Marceglia, S.; Servello, D.; Foffani, G.; Porta, M.; Sassi, M.; Mrakic-Sposta, S.; Rosa, M.; Barbieri, S.; Priori, A. Thalamic single-unit and local field potential activity in Tourette syndrome. Mov. Disord. 2010, 25, 300-308. [CrossRef]

32. Zhuang, P.; Li, Y.; Hallett, M. Neuronal activity in the basal ganglia and thalamus in patients with dystonia. Clin. Neurophysiol. 2004, 115, 2542-2557. [CrossRef]

33. Alam, M.; Schwabe, K.; Lütjens, G.; Capelle, H.H.; Manu, M.; von Wrangel, C.; Müller-Vahl, K.; Schrader, C.; Scheinichen, D.; Blahak, C.; et al. Comparative characterization of single cell activity in the globus pallidus internus of patients with dystonia or Tourette syndrome. J. Neural Transm. (Vienna) 2015, 122, 687-699. [CrossRef] [PubMed]

34. Malekmohammadi, M.; Sparks, H.; AuYong, N.; Hudson, A.; Pouratian, N. Propofol Anesthesia Precludes LFP-Based Functional Mapping of Pallidum during DBS Implantation. Stereotact. Funct. Neurosurg. 2018, 96, 249-258. [CrossRef]

35. Tsai, S.T.; Chen, T.Y.; Lin, S.H.; Chen, S.Y. Five-Year Clinical Outcomes of Local versus General Anesthesia Deep Brain Stimulation for Parkinson's Disease. Parkinsons Dis. 2019, 17. [CrossRef]

36. Harris, K.D.; Henze, D.A.; Csicsvari, J.; Hirase, H.; Buzsáki, G. Accuracy of tetrode spike separation as determined by simultaneous intracellular and extracellular measurements. J. Neurophysiol. 2000, 84, 401-414. [CrossRef]

37. Henze, D.A.; Borhegyi, Z.; Csicsvari, J.; Mamiya, A.; Harris, K.D.; Buzsáki, G. Intracellular features predicted by extracellular recordings in the hippocampus in vivo. J. Neurophysiol. 2000, 84, 390-400. [CrossRef]

38. Marsden, J.E.; Tromba, A.J. Vector Calculus, 5th ed.; Freeman: New York, NY, USA, 2003.

39. Peña, D. Análisis de Datos Multivariantes; McGraw-Hill/Interamericana de España: Madrid, Spain, 2002.

40. McLachlan, G. Discriminant Analysis and Statistical Pattern Recognition; Wiley: Hoboken, NJ, USA, 2004.

41. Seifried, C.; Weise, L.; Hartmann, R.; Gasser, T.; Baudrexel, S.; Szelényi, A.; van de Loo, S.; Steinmetz, H.; Seifert, V.; Roeper, J.; et al. Intraoperative microelectrode recording for the delineation of subthalamic nucleus topography in Parkinson's disease. Brain Stimul. 2012, 5, 378-387. [CrossRef]

42. Li, X.; Zhuang, P.; Hallett, M.; Zhang, Y.; Li, J.; Li, Y. Subthalamic oscillatory activity in parkinsonian patients with off-period dystonia. Acta Neurol. Scand. 2016, 134, 327-338. [CrossRef]

43. Möttönen, T.; Katisko, J.; Haapasalo, J.; Tähtinen, T.; Saastamoinen, A.; Peltola, J.; Öhman, J.; Lehtimäki, K. The Correlation between Intraoperative Microelectrode Recording and 3-Tesla MRI in Patients Undergoing ANT-DBS for Refractory Epilepsy. Stereotact. Funct. Neurosurg. 2016, 94, 86-92. [CrossRef]

44. Alam, M.; Sanghera, M.K.; Schwabe, K.; Lütjens, G.; Jin, X.; Song, J.; von Wrangel, C.; Stewart, R.M.; Jankovic, J.; Grossman, R.G.; et al. Globus pallidus internus neuronal activity: A comparative study of linear and non-linear features in patients with dystonia or Parkinson's disease. J. Neural Transm. (Vienna) 2016, 123, 231-240. [CrossRef] [PubMed]

45. Chen, T.; Mirzadeh, Z.; Chapple, K.M.; Lambert, M.; Evidente, V.G.H.; Moguel-Cobos, G.; Oravivattanakul, S.; Mahant, P.; Ponce, F.A. Intraoperative test stimulation versus stereotactic accuracy as a surgical end point: A comparison of essential tremor outcomes after ventral intermediate nucleus deep brain stimulation. J. Neurosurg. 2018, 129, 290-298. [CrossRef] [PubMed]

46. Brahimaj, B.; Kochanski, R.B.; Sani, S. Microelectrode accuracy in deep brain stimulation surgery. J. Clin. Neurosci. 2018, 50, 58-61. [CrossRef] [PubMed]

(C) 2019 by the authors. Licensee MDPI, Basel, Switzerland. This article is an open access article distributed under the terms and conditions of the Creative Commons Attribution (CC BY) license (http://creativecommons.org/licenses/by/4.0/). 